\title{
Diagnóstico de dificultades lingüísticas en producciones escritas en español de inmigrantes marroquíes residentes en España
}

\author{
Cristina Mata Verdoy y Anna Doquin de Saint Preux
}

Este trabajo propone un diagnóstico de las principales dificultades, para el aprendizaje de la lengua española, de inmigrantes marroquíes de la comunidad de Madrid, aspirantes a la obtención del Diploma LETRA, a partir de la descripción, clasificación y análisis de los errores cometidos en sus producciones escritas de los exámenes previstos para la obtención del citado Diploma. Se clasifican los datos obtenidos conforme al modelo de Fernández (1997), con algunas modificaciones que se consideran impuestas por la frecuencia y cualidad de determinado grupo de errores. A partir de ello, se intenta aportar una descripción de su interlengua desde la consideración de tres sistemas: el de la lengua materna, el de la lengua francesa y el de la lengua española. Para ilustrar estos criterios, hemos aportado opiniones de autores, que nos han parecido oportunas, tanto para afianzar nuestras conclusiones como para anotar aquellas que no se han visto confirmadas por los resultados obtenidos.

Palabras claves: Análisis contrastivo, Análisis de errores, Inmigrantes marroquíes, Español como Lengua de Migración, Interlengua, Transferencia, Prosodia.

Diagnosis of linguistic difficulties in written language production in Spanish of Moroccan resident in Spain inmigrants. This paper aims to diagnose the main difficulties that Moroccan immigrants face when aspiring to obtain the LETRA Diploma in the Autonomous Community of Madrid. It does this by describing, classifying and analysing the errors made in the written exams required to obtain the aforementioned certificate. The collected data are classified according to Fernandez' model (1997), with certain modifications imposed by the frequency and quality of certain errors. From them, this study attempts to describe the stage of their interlanguage by taking three language systems into consideration: that of the 
Mother Tongue (L1); that of the French Language (L2); and that of the Spanish Language (L3). In order to illustrate the criteria, opinions of other authors that have been considered appropriate have been provided, both to confirm the opinions expressed herein and to present opinions that have not been confirmed by the results obtained.

Keywords: Contrastive analysis, Analysis of errors, Moroccan Inmigrants, Spanish as a Language of Migration, Interlanguage, Transfer, Prosody.

\section{Introducción}

Este estudio se enmarca dentro de un proyecto de investigación cuya finalidad es la integración lingüística de la población inmigrante de la comunidad de Madrid. El proyecto INMIGRA, financiado por la Comunidad de Madrid y los Fondos Europeos, busca facilitar esa integración y la acogida de la población inmigrante de la Comunidad de Madrid mediante un proyecto pluridisciplinar titulado "La población migrante de la comunidad de Madrid: estudio multidisciplinar y herramientas para la integración sociolingüística”. En una fase anterior del proyecto, que se desarrolló entre 2007 y 2010, se diseñó e implementó un examen de certificación denominado "Diploma LETRA" (Lengua Española para Trabajadores Inmigrantes), dirigido a evaluar la competencia comunicativa de inmigrantes adultos, en los ámbitos administrativos y laborales. Este examen consta de cuatro pruebas: comprensión lectora, comprensión audiovisual, expresión e interacción escrita y expresión e interacción oral, y certifica un nivel inicial A. El presente estudio se incluye en la tercera fase del proyecto INMIGRA (INMIGRA3_CM. H2019/HUM-5772). En esta fase del proyecto, se pretende analizar el corpus obtenido de las 5 convocatorias del Diploma LETRA con el fin de detectar y analizar las dificultades específicas de la población inmigrante y de cada comunidad lingüística para proponer soluciones e indagar los procesos de adquisición de la lengua. En este marco, nuestro estudio intenta establecer un diagnóstico de las dificultades lingüísticas a las que se enfrentan los inmigrantes marroquíes que residen en la Comunidad de Madrid a la hora de escribir un texto en español. Realizamos este diagnóstico a través del análisis de los errores cometidos en la prueba de expresión e interacción escrita del diploma LETRA (dos tareas) de una muestra de candidatos marroquíes que comparten las mismas características lingüísticas (L1 árabe y L2 francés). 


\section{Estado de la cuestión}

Los estudios previos sobre las dificultades de aprendientes marroquíes en español insisten todos en el hecho de que para ellos la lengua española no suele ser su L2 sino más bien su L3 o L4, debido a que la sociedad marroquí se caracteriza por el plurilingüismo. Por lo tanto, las posibles influencias no solo provienen de su lengua materna (árabe o dariya) sino también de otras lenguas que hablan (bereber o francés, en la mayoría de los casos). Estudios como los de Al-Madkouri (1995), Benyaya (2007) o Ammadi (2009) destacaron las principales dificultades en español así como las interferencias fonológicas, morfosintácticas y léxicas en sus producciones lingüísticas.

Al-Madkouri (1995) estudió las dificultades en español en la interlengua de inmigrantes marroquíes residentes en España. ${ }^{1}$ Afirma que su interlengua se puede caracterizar por rasgos fonéticos, fonológicos y sintácticos que la lengua nativa impone a la adquirida. Al-Madkouri incide en la dificultad que representa para ellos el sistema vocálico del español. En este caso, el análisis contrastivo entre el sistema vocálico del árabe y del español explica esa dificultad: el árabe o el dariya (árabe marroquí) cuentan con una vocal abierta, baja central /a/ y dos vocales cerradas, altas: una anterior /i/ y otra posterior /u/. El sistema vocálico del español cuenta con dos vocales más, de abertura media; una anterior /e/ y otra posterior /o/. Por tanto, en español se dan dos pares de oposiciones: una en la zona palatal /e/, /i/ y otra en la velar /o/, /u/, que no existen en el sistema vocálico del árabe. Señala Madkouri que "es difícil a veces saber si los hablantes se refieren a "piso" o a "peso", a "muro" o a "moro", en un fenómeno de neutralización. En cuanto al consonantismo, los alumnos marroquíes pronuncian las consonantes más cerradas, debido a que el árabe o dariya es una lengua consonántica. Destaca también el fenómeno del seseo y las vacilaciones entre las consonantes $/ \mathrm{s} /, / \theta /$, por influencia del francés esta vez, según Madkouri. Unido a las oposiciones vocálicas anteriormente señaladas, contribuye al aumento de las dificultades o posibles confusiones pues, como dice Madkouri, podemos dudar de "si el hablante se refiere a" puso", "poso" o "pozo"”. Otro aspecto diferencial importante es el de las relaciones morfosintácticas pues, tanto en el caso del árabe como en el bereber, la relación entre los constituyentes de la oración es más semántica que sintáctica, es decir, que la semántica es la que establece la relación entre los elementos y las categorías gramaticales y no la sintaxis. Son habituales, en las construcciones en español de los arabófonos: 1) la falta de los conectores internos: preposiciones, adverbios..., 2) los alargamientos de la frase (frases párrafo) mediante reiteraciones de conectores, por hipercorrección. Es 
común la repetición de partículas tales como: pues, y, o el uso de sin embargo, no obstante, pero... sin que posean su valor exacto. Otra de las principales dificultades con que se encuentra el alumno marroquí, estudiante de español, es la flexión verbal. El dariya dispone de sólo 19 formas morfológicas del verbo frente a 115 de la lengua española. Además, en aquella lengua, el tiempo tiene un carácter contextual, mientras que en español es morfológico. Una forma verbal como $\mathrm{Bqa}$, en árabe marroquí, señala Madkouri, no significa que está en pasado, sino que lo puede estar también en presente. Los tiempos del subjuntivo en español (muy al contrario de la concepción árabe) no son tiempos de acción sino tiempos relacionales del verbo. No se refieren a la ubicación de una determinada acción en el eje temporal absoluto, sino a su localización real o ficticia en correspondencia con otras acciones. En suma, las diferencias en cuanto al tiempo verbal entre el árabe y el español se pueden sintetizar en los puntos siguientes: 1) diferencia entre el punto de vista y el enfoque de la acción del verbo, 2) diferencia entre la consideración del tiempo objetivo de la acción del verbo y la del momento del habla, 3) la escasa coincidencia entre los factores que condicionan el régimen del modo. En el caso de marroquíes con conocimientos de francés, la transferencia positiva puede representar una cierta ventaja: tanto para la adquisición del sistema verbo temporal como para la sintaxis. La interlengua de los aprendientes marroquíes presenta frecuentemente procedimientos propios de la lengua francesa aplicados al español y numerosos fenómenos de interferencia. Según Madkouri, por ejemplo, las incorrecciones en el empleo de las preposiciones se deben más a la influencia del francés que del árabe.

Benyaya (2007) se centra en los aspectos lingüísticos del español más difíciles de adquirir para alumnos marroquíes escolarizados en la secundaria de su país. ${ }^{2}$ Revisa los aspectos fonológicos, morfosintácticos y léxico-semánticos, comparándolos con las respectivas estructuras del árabe. El corpus lo conforma Benyaya mediante grabaciones tomadas a alumnos de institutos públicos de distintas ciudades y regiones de Marruecos, de edades entre 16 y 18 años, pertenecientes a los niveles elemental, medio y avanzado del Ciclo de Cualificación y a primero y segundo de Bachillerato. Afirma Benyaya que, en el plano fonológico, la riqueza consonántica del árabe supone una ventaja para el estudiante marroquí ya que esto facilita la pronunciación del español, incluso la de los sonidos inexistentes en el árabe clásico. En cuanto al vocalismo, podría repetirse lo dicho a propósito del estudio de Madkouri sobre las dificultades que el hablante marroquí encuentra con las vocales medias. Benyaya habla de fenómenos de asimilación, poniendo ejemplos como

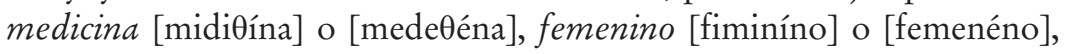
dinero [denéro] o [diníro]. También comenta que las vocales experimen- 
tan un fenómeno de abertura en contacto con las consonantes faríngeas y uvulares: averigüéis se realiza como [averigwáis]. Los diptongos plantean también problemas al hablante marroquí, ya que este tipo de combinación vocálica es inexistente en árabe. Por ello, aparece una tendencia a simplificarlos o a sustituir las vocales formantes por otras. Sobre todo, existe una tendencia general a reducir el diptongo creciente [je] eliminando, en la mayoría de las veces, la semiconsonante [j]: empiezan [empésan], empieza [empí日a], fiesta [fésta], hierro [éro]. También se reducen los hiatos: maestro [mástro], preocupes [prekópes]. La confusión vocálica puede dar lugar a fenómenos como la pronunciación de un hiato en diptongo, o justamente al proceso inverso, es decir, a la ruptura de un diptongo convirtiéndolo en hiato: aeropuerto [airopwérto], realidad [rjalidád], bucear [böjár], mediterráneo [miditránjo]. En cuanto al consonantismo, la oposición $/ \mathrm{b} /, / \mathrm{p} /$ no plantea casi nunca problemas, debido a que, aunque la oclusiva sorda es inexistente en el árabe clásico, suele ser conocida gracias al estudio del francés. La palatal nasal $/ \mathrm{n} /$, al ser desconocida en árabe, suele representar una dificultad. El alumno sólo le encuentra referencia en la analogía, descomponiendo el sonido y realizándolo como $n+i$, porque es la pronunciación que más se acerca a su percepción del sonido: niño [nénjo], mañana [manjána]. En la escritura, las grafías que representan a dos o más fonemas son muy conflictivas. Es el caso de la letra "c", cuya ambivalencia para representar a la vez el sonido $[\mathrm{k}]$ y $[\theta]$ da lugar a muchas confusiones: "almuerco (por almuerzo). El acento gráfico supone asimismo una importante traba, ya no solo por el desconocimiento de las reglas de acentuación, sino que el alumno, al tener dificultades para percibir auditivamente la carga fonética, y por tanto, para localizar la posición del acento tónico dentro de la palabra, a la hora de escribir, lo plasma -o no- de forma aleatoria, sin aplicar más criterio que el de la simple intuición.

Ammadi (2009)3 ${ }^{3}$ establece las dificultades específicas en la enseñanza de español a aprendientes marroquíes de forma general. Habla del descuido del alumnado arabófono en cuanto a la puntuación y la ortografía que, por conservadurismo con los hábitos del árabe, las suple mediante el uso de comillas. Subraya también el obstáculo de la morfosintaxis: dado que la estructura del árabe es verbo + sujeto, tiende a mantenerla, en contra de la de sujeto + predicado, propia del español. Otros aspectos señalados por Ammadi son la tendencia a las construcciones ilativas mediante $y$ polisindética, el alargamiento de la frase mediante la aposición y el uso del gerundio. Asimismo, destaca la falta de concordancia en género, número y persona (ya que las normas de concordancia en árabe son distintas), la confusión en la asimilación de los pronombres personales, la ausencia del verbo copulativo (por no figurar éste en el árabe) y las dificultades relacionadas con el uso de los verbos reflexivos. 
En el caso de nuestro estudio, los informantes son todos adultos, de nacionalidad marroquí, que residen en España y que declaran hablar francés además de árabe marroquí o dariya. Santos de la Rosa (2014) explica que no es fácil detectar el origen de la transferencia en caso de aprendientes marroquíes, pues esta puede deberse tanto a la lengua estándar (árabe) como a su LM (árabe dialectal) o a la lengua extranjera L2 (francés) que haya podido servir como enlace intermedio. Esto supone una llamada de atención para el propósito de nuestro trabajo, dificultad que trataremos de resolver intentando deslindar las transferencias del francés de las demás recurriendo, en primer lugar, a las relaciones de errores ofrecidas en investigaciones sobre estudiantes arabófonos de ELE; en segundo lugar, al análisis contrastivo de los sistemas de las lenguas francesa y española y, en tercer lugar, tomando en consideración las conclusiones de los estudios sobre estudiantes franceses de ELE, tales como los de Sáez (2012) y Doquin de Saint Preux y Sáez (2014).

\section{Estudio empírico}

\subsection{Objetivos y metodología de investigación}

Como señalamos anteriormente, el objetivo de este estudio es diagnosticar cuáles son los errores de carácter ortográfico, gráfico, léxico, morfosintáctico y estructural más comunes en las producciones escritas de los nativos marroquíes candidatos al Diploma LETRA. Llevamos a cabo una investigación de carácter heurístico y descriptivo para determinar las principales dificultades que podían encontrar a la hora de escribir textos en español. Se recogieron todos los errores detectados en los textos para clasificarlos y cuantificarlos.

\subsection{Descripción de la muestra y de los informantes}

Disponemos, para nuestro estudio, de 40 muestras escritas, pertenecientes a 20 informantes marroquíes, inmigrantes, que se presentaron a la convocatoria 4 del Diploma LETRA en 2014. Son adultos de ambos sexos, que declaran haber tenido una escolarización en francés, sin precisión del nivel adquirido en esta lengua. El francés es materia obligatoria en el sistema educativo de Marruecos, en la Enseñanza Básica Obligatoria, desde los 8 a los 15 años de edad. También es obligatorio en todos los cursos de la Enseñanza Secundaria General (Berengueras 2013). Consideramos esta lengua como su L2. Sus lenguas maternas son el árabe o dariya (dialecto árabe marroquí) y/o el amazig (bereber). En 
el dariya, en su forma hablada, se da una fuerte diferenciación entre el sociolecto bajo y el sociolecto alto, este considerado como forma dialectal de prestigio: una especie de lengua vehicular que se está imponiendo al resto del país y cuya base es el dialecto hablado en Casablanca y Rabat, las capitales, una comercial y la otra política (Moscoso 2003:168-169 y 176).

Dado que no hemos tenido acceso a una mayor información sobre los informantes, debemos señalar algunos aspectos para no desvirtuar el auténtico valor de nuestro estudio pues, realmente, no sabemos la edad ni cuál es el nivel de estudios de cada informante (lo reconocemos diverso), tampoco si su lengua materna puede haber sido árabe marroquí (dariya) o amazig. Desconocemos también cuál es su competencia en francés, considerada su L2. Por tanto, nuestras conclusiones no podrán ser valoradas como caracterizadoras de un determinado nivel de interlengua que pudiera entenderse extrapolable. Pretendemos, no obstante, poner de manifiesto cuál es el panorama, en cuanto a capacidad de integración lingüística en nuestro idioma, de esta población de inmigrantes marroquíes y detectar las principales dificultades que encuentran en orden a poder plantear una metodología para favorecer dicha integración.

\subsection{Instrumentos de recogida de datos}

Disponemos, por cada uno de los informantes, de dos pruebas de expresión e interacción escrita (las tareas 2 y 3 de la prueba, que identificamos como tarea A y tarea B respectivamente. La tarea 1 es de expresión oral, por lo que su condición no es objeto de este estudio). En la tarea $\mathrm{A}$, se ha pedido a los aspirantes que formulen un anuncio con el que se ofrezcan para desempeñar un trabajo y en donde deben especificar: 1) qué trabajo saben hacer, 2) cuándo podrían hacerlo, 3) cuánto cobran por su trabajo, 4) forma de contacto. En la tarea B, se les ha proporcionado un correo electrónico, supuestamente dirigido al examinando por la coordinadora de actividades de un centro cultural, con el que se les invita a asistir a la fiesta de fin de curso y a participar con alguna aportación en las actividades diversas que se organizan. Deben dar respuesta de confirmación de asistencia y actividad con la que desean participar.

\subsection{Análisis de datos y métodos de clasificación}

Para llevar a cabo el análisis de errores (AE), fue necesario realizar una clasificación de los mismos y establecer tipologías mediante un proce- 
so que debe pasar por las fases siguientes: a) detección de los errores b) agrupamiento de los mismos conforme a la norma lingüística, c) explicación del posible origen y causas que hayan podido contribuir en su producción. Entre las tipologías más usadas para el AE en ELE se encuentran las de Isabel Santos (1993), Sonsoles Fernández (1997) y Graciela Vázquez (1999). Para este estudio nos pareció que el criterio de clasificación de los errores más ad hoc con el trabajo que nos planteamos es el de Fernández (1997), en el que introducimos alguna modificación. En el primer criterio, llamado "errores léxicos por la forma", se presentó el problema de incluir o no entre ellos las lexías en cuya forma de escritura se detectara cualquier tipo de error pero, dado que la mayoría de esas palabras lexicales contenían errores achacables fundamentalmente al vocalismo o consonantismo, nos pareció útil considerarlas como errores gráficos, que Fernández incluye en el apartado de "errores gráficos". Por otra parte, dado que los errores ortográficos están omnipresentes en las muestras, les hemos dedicado un apartado específico. Así pues, entre los errores léxicos debidos a la forma, incluimos aquellos términos cuyo error consiste en lo que tradicionalmente se viene denominando como "errores esporádicos", bien por adición: prótesis, epéntesis y paragoge o bien por supresión: aféresis, síncopa y apócope. En tal caso, nos ha parecido coherente incluir también el fenómeno de la metátesis.

\subsection{Resultados}

Hemos recogido los resultados en la tabla 1, que reproducimos a continuación. Se han detectado 428 errores (no contabilizamos los signos de puntuación). En la primera columna (GRUPO) señalamos el tipo o grupo de errores, en la segunda (TIPO) describimos el error que contabilizamos, en la tercera $\left(\mathrm{N}^{\circ}\right)$ anotamos el número de errores advertidos (si un mismo informante comete varias veces un mismo error, sólo se contabiliza una vez), en la cuarta (\% Total) anotamos el porcentaje del error sobre los 428 totales, aproximándolo por defecto. 


\begin{tabular}{|c|c|c|c|}
\hline GRUPO & TIPO & $\mathbf{N}^{0}$ & $\%$ (Total) \\
\hline \multirow[b]{4}{*}{ Errores gramaticales } & Concordancia en el S. N. & 9 & 2.10 \\
\hline & Determinantes & 21 & 4,90 \\
\hline & Verbos ser/estar & 1 & 0,23 \\
\hline & Conjugación verbal & 31 & 7,24 \\
\hline \multirow{4}{*}{$101(23,59 \%)$} & Pronombres & 7 & 1,63 \\
\hline & Preposiciones & 23 & 5,37 \\
\hline & Adverbios & 0 & 0,00 \\
\hline & Enlaces oracionales: coordinantes/subordinantes & 9 & 2,10 \\
\hline \multirow{2}{*}{$\begin{array}{l}\text { Errores léxicos } \\
33(7,71 \%)\end{array}$} & Por confusión en el significado & 18 & 4,20 \\
\hline & Por errores de forma (metaplasmos) & 15 & 3,50 \\
\hline \multirow{3}{*}{$\begin{array}{l}\text { Errores gráficos } \\
\text { Vocales/consonantes } \\
78(18,22 \%)\end{array}$} & En vocales & 35 & 8,17 \\
\hline & En diptongos/hiatos & 21 & 4,90 \\
\hline & En consonantes & 22 & 5,14 \\
\hline \multirow{4}{*}{$\begin{array}{l}\text { Errores ortográficos } \\
191(44,64 \%)\end{array}$} & En tildes & 66 & 15,42 \\
\hline & Confusiones mayúscula/minúscula & 101 & 23,59 \\
\hline & Oposición de pares de letras & 12 & 2,80 \\
\hline & Separación/unión de palabras & 12 & 2,80 \\
\hline \multirow{2}{*}{$\begin{array}{l}\text { Errores discursivos } \\
25(5,84 \%)\end{array}$} & Incoherencias discursivas & 4 & 0,93 \\
\hline & Construcciones agramaticales & 21 & 4,90 \\
\hline
\end{tabular}

Tabla 1. Resumen general de los datos cuantitativos de errores detectados

Como podemos comprobar, la ortografía representa la principal dificultad en los textos analizados (191 errores). La confusión en el empleo de mayúsculas/minúsculas arroja más de la mitad de los errores de este grupo $(52,87 \%)$, lo que deja bastante claro que este aspecto presenta una de las mayores dificultades para nuestros informantes (ya hemos señalado que no contabilizamos los signos de puntuación, de los que hemos detectado aproximadamente 180). Se confirma con ello la opinión de Ammadi (2009) en cuanto a los descuidos de los informantes respecto a la puntuación; en su opinión, por conservadurismo con los hábitos del árabe. Suponen casi la mitad del total de errores (44,64\%). En segundo lugar tenemos los errores gramaticales: 101 (23,59\%). Como vemos, el rango cuantitativo lo encabezan los errores en el sistema verbal (31) (a los que habría que sumar el error en ser/estar), seguido de las preposiciones (23) y los determinantes (21). Este orden es el mismo que habían presentado Sáez (2012) y Doquin de Saint Preux y Sáez (2014) en sus estudios sobre los errores de franceses en español, lo que viene a demostrar que el rango de dificultad que encuentran los marroquíes francófonos para el aprendizaje de las categorías gramaticales del español es parecido al que encuentran los franceses. El vocalismo y consonantismo contabilizan 78 errores $(18,22 \%)$. Si agrupamos los errores de los diptongos con los vocálicos, arrojan una suma de 56, lo que supone el $71,79 \%$ del total de errores gráficos: una notable diferencia sobre los errores cometidos en las consonantes (28,20\%); prueba evidente de que 
los aprendientes marroquíes manifiestan tener serios problemas con el vocalismo del sistema español pero no tanto con el consonántico. Esto confirma los resultados de los estudios anteriores sobre la adquisición del español en Al-Madkouri (1955), Benyaya (2007), Ammadi (2009).

\subsubsection{Errores más significativos}

De la lectura de nuestros resultados se deduce que las principales dificultades en la tarea de escritura, según se manifiestan en las muestras escritas analizadas, son: 1) dificultades ortográficas (uso de la tilde y alternancia mayúscula/minúscula), 2) uso de la conjugación verbal y 3 ) la alternancia vocálica.

\subsubsection{Dificultades ortográficas}

Los inmigrantes marroquíes suelen encontrar dificultades en el uso de mayúsculas y acentos. En la escritura árabe no existe la doble grafía mayúscula/minúscula de las letras por lo que esta dualidad, común al francés y al español, resulta extraña para los marroquíes que se inician en la escritura de estas lenguas. A la dificultad de tener que establecer la correspondencia entre el alifato y el abecedario latino, más la que supone el cambio de linealidad (de izquierda a derecha), se une la de un doble grafismo que, además, está sometido a unas reglas ortográficas fijas. La ortografía de uso de las letras mayúsculas en francés y español es prácticamente la misma. Este error nos indica que, aunque nuestros informantes tienen conocimientos de francés, no necesariamente están acostumbrados a escribir en esa lengua.

Otra dificultad recurrente es el uso de los acentos. La tilde española tiene la función de marcar el acento de intensidad sobre la sílaba en que aparece, mientras que en francés todas las palabras son consideradas fonéticamente agudas y el acento indica la abertura con que ha de pronunciarse la vocal afectada. La tilde, en español, es un elemento fonológico, distintivo, suprasegmental. Este aspecto no es del todo extraño para el árabe, que lo suple por la cantidad o duración. Al-Zawan (2005:74) nos informa de cómo en la lengua árabe se recurre al doble sonido vocálico para marcar la intensidad entonativa, distinguiendo entre sonidos vocálicos simples y sonidos vocálicos prolongados. Así /a:/ = aa, /i:/ = ii , /u:/ = uu, lo que permite distinguir entre yamal (camello) y yamaal (belleza), por ejemplo. Este fenómeno de duplicación, también se da en las consonantes, con valores distintivos al igual que con las vocales. Esta variedad entonativa de las palabras en español exige, para la escritura, una normativa ortográfica sobre el uso de la tilde, de indudable dificultad para el nivel de clasificación de la prueba. No nos 
sorprende, pues, que hayamos detectado, del total de errores ortográficos, un $34,02 \%$ achacable a la tilde. Para Benyaya (2007), el acento gráfico supone una importante traba para los aprendientes marroquíes; en primer lugar, por el desconocimiento de las reglas de acentuación y en segundo, por tener dificultades para percibir auditivamente la carga fonética y, por tanto, para localizar la posición del acento tónico dentro de la palabra. Por eso, a la hora de escribir, si ponen acento, lo sitúan de forma aleatoria, sin aplicar más criterio que el de la simple intuición. A veces pudiera llegar al extremo de llevar al informante a considerar que las funciones del acento en francés y español son comparables: un caso de transferencia negativa, como se demuestra por algunos ejemplos de las muestras, que nos parecen claramente indiciarios de lo que acabamos de afirmar ("téléfono, "dispuesto à trabajar, "vamos à traer).

\subsubsection{Dificultades con el sistema verbal español}

El primer comentario que sugiere la situación del empleo de los tiempos por parte de nuestros informantes es habernos encontrado una gran variabilidad:

\begin{tabular}{|l|l|}
\hline Infinitivos & 38 \\
\hline Imperativos & 3 \\
\hline Formas de presente de indicativo & 88 \\
\hline Formas en futuro simple & 5 \\
\hline Formas de perfecto compuesto & 3 \\
\hline Formas de futuro compuesto & 1 \\
\hline Formas de presente de subjuntivo & 6 \\
\hline Formas de condicional simple & 4 \\
\hline Perífrasis con infinitivo & 43 \\
\hline Perífrasis con gerundio & 2 \\
\hline TOTAL & 193 \\
\hline
\end{tabular}

Tabla 2. Distribución de tiempos y formas verbales encontrados en las muestras

Podemos subrayar también que muchos usos de los tiempos verbales son adecuados, seguramente por transferencia positiva del francés, que posee un sistema similar.

Por su interés relevante, adjuntamos la tabla 3 , en la que hemos recogido la relación de errores. 


\begin{tabular}{|c|c|c|}
\hline & ERROR & CORRECCIÓN \\
\hline \multirow{23}{*}{$\begin{array}{l}\text { Tiempo, } \\
\text { Modo y } \\
\text { Aspecto. }\end{array}$} & 6.1. necesita & necesite \\
\hline & 11.1. sabo & sé \\
\hline & 11.2. he recibidomos & hemos recibido \\
\hline & 11.2. si me gustamos iremos. & Sí, me gustaría ir \\
\hline & 11.2. de iremos & de ir \\
\hline & 11.2. estamos allí & estaremos allí \\
\hline & 14.2. Hi enviados & He enviado \\
\hline & 28.1. HABLAMOS & hablemos \\
\hline & 28.2. ABER EL ANIO CI VIENE & a ver el año que viene \\
\hline & 28.2. MECANA VINER & me encantaría venir \\
\hline & 53.2. pasarais & paséis \\
\hline & 62.2. traigo & llevaré \\
\hline & 63.2. gusta & gustaría \\
\hline & 63.2. preparé & prepararé \\
\hline & 63.2 traego & traeré \\
\hline & 64.1. podría trabajo & podría trabajar \\
\hline & 64.1 pregonta & preguntar \\
\hline & 65.1. yo sabe hacer & yo sé hacer \\
\hline & 68.1 freir pescados & frío pescado \\
\hline & 68.2. cada persona que traer los pasteles & que cada persona traiga los pasteles \\
\hline & 63.1. ese trabajo cobra $1000 €$ & por ese trabajo cobro $1000 €$ \\
\hline & 77.1. puedo como ayudante & puedo trabajar como ayudante \\
\hline & 79.1. coboro & cobraba \\
\hline \multirow{7}{*}{$\begin{array}{l}\text { Número } \\
\text { y } \\
\text { persona }\end{array}$} & 7.2. ha recibido & he recibido \\
\hline & 14.2. sabe & sé \\
\hline & 28.2. ME ENBETAR & invitarme \\
\hline & 53.2. usted me has envitado & Usted me ha invitado \\
\hline & 65.1 yo sabe & Yo sé \\
\hline & 77.1. (yo) puede hacerlo & Yo puedo hacerlo \\
\hline & 77.2. no puede estar & no puedo estar \\
\hline
\end{tabular}

Tabla 3. Errores en el empleo de las formas verbales

En la tabla 4, reflejamos la síntesis de errores detectados.

\begin{tabular}{|l|r|}
\hline Formas de presente de indicativo por otros tiempos verbales & 10 \\
\hline Tiempos de indicativo por subjuntivo & 3 \\
\hline Formas de infinitivo por otros tiempos verbales & 2 \\
\hline Formas morfológicamente mal construidas & 8 \\
\hline TOTAL & 23 \\
\hline
\end{tabular}

Tabla 4. Errores más frecuentes detectados en el uso de los tiempos verbales

La tipología de los textos exigidos para sendas pruebas, es evidente que no obliga al empleo de tiempos del pasado y sí al uso del presente. Dato a tener en cuenta a la hora de valorar los resultados: 
La mayor parte de esos errores son debidos al empleo del presente en vez del futuro o condicional, tiempos que plantean serias dificultades a los arabófonos. El condicional, además de su carácter hipotético, tiene un matiz de antefuturo. Por otra parte, en francés, las formas de primera persona del singular del futuro y condicional son homófonas: Je serais/je serai. En el total del corpus, sólo encontramos seis formas de subjuntivo, todas en presente, menos en un informante que utiliza una forma de imperfecto, "pasarais. Esta es la única constitutiva de error, consistente en el uso de esta forma en vez de su correlativa del presente paséis. Anotamos también tres empleos de indicativo por subjuntivo: necesita por necesite, hablamos por hablemos, y "cada persona que traer por que cada persona traiga. Con esta situación podría deducirse que el subjuntivo no está bien asimilado por los informantes y o bien lo evitan o bien lo usan como el indicativo. Ya se ha referido por distintos autores la mayor riqueza del español con respecto a la lengua árabe en cuanto a modos y tiempos verbales. Uno de los aspectos que ha merecido mayor atención por parte de los investigadores es la formación de las oposiciones pasado/futuro y la más sutil del aspecto perfectivo/imperfectivo. Según Al-Zawan (2005: 293) la cuestión del tiempo, en árabe, es un problema de aspecto y su formación se realiza mediante partículas y sufijos. La información que transmiten las formas de perífrasis verbal en español no se limita al carácter imperfectivo/perfectivo. Existen otras relaciones aspectuales tales como, el carácter incoativo, iterativo o durativo. A esto tenemos que añadir los valores modales de obligación, voluntad y posibilidad. Veamos el resultado con algunas de las perífrasis halladas en las muestras:

\begin{tabular}{|l|l|r|}
\hline Perífrasis de posibilidad y voluntad & Con auxiliar poder & 20 \\
\cline { 2 - 3 } & Con auxiliar querer & 1 \\
\cline { 2 - 3 } & Con auxiliar tener que & 1 \\
\hline Perífrasis incoativas & Con auxiliar ir $+a$ & 16 \\
\hline
\end{tabular}

Tabla 5. Valor aspectual de las perífrasis halladas en las muestras

La mayor parte de las perífrasis de posibilidad y voluntad están construidas con el auxiliar en presente, menos dos casos en condicional. Las 16 formas incoativas van en presente más preposición a más infinitivo. Este panorama demuestra un cierto dominio de estas fórmulas, pues debemos señalar que la mayor parte están construidas correctamente. Entre las incoativas, singularizamos dos: la expresión "si voy ir en fiesta de curso" y "no voy estar con vosotros", por el hecho de que entre el verbo auxiliar y el infinitivo no han escrito la preposición $a$. Las perífrasis incoativas del español con ir+a+infinitivo se corresponden con el futur proche del francés, que se construye con aller+infinitif. Esperábamos encontrar un mayor número de casos de perífrasis incoativas con ausencia de preposición por interferencia 
del francés pero los escritos demuestran que la fórmula del español es perfectamente asimilable por los hablantes marroquíes, capaces, en su caso, de establecer las diferencias contrastivas resultantes del francés y español.

\subsubsection{Dificultades gráficas en el vocalismo}

En las muestras escritas, se encontraron numerosos errores gráficos en las vocales. Estos errores apoyan las teorías de Al-Madkouri (1995) y Benyaya (2007), según las cuales los hablantes marroquíes tienen dificultades fundamentalmente para la distinción /e/, /i/ y, en menor medi$\mathrm{da}, / \mathrm{o} /, / \mathrm{u} /$. Según Benyaya, la tendencia es a homogeneizar las vocales, siendo normalmente la primera vocal la que influye sobre la segunda, en un proceso de asimilación (A). Sin embargo, no es exactamente eso lo que hemos podido constatar en nuestras muestras, pues los fenómenos de disimilación (D) son numerosos. En la tabla 6 podemos ver todos los errores recogidos en la realización de vocales, diptongos e hiatos.

Hamparzoumian y Barquín (2005:34), en un estudio con niños marroquíes escolarizados en Andalucía, hablan del sistema vocálico marroquí, definiéndolo como más complejo que el español. Para sostener tal afirmación, hemos de entender, lógicamente, que estos autores adoptan un criterio fonético, no fonológico. Sin embargo, al pretender explicar la naturaleza de los errores, lo hacen con un criterio fonológico:

En el sistema vocálico marroquí existen las cinco vocales españolas y, al menos, dos intermedias: /a/, /./, /e/, /'// / /, /o/, /u/ que se producen por diferencias en la cantidad, o longitud vocálica. La longitud y el timbre interactúan y producen unas articulaciones que varían, en función del énfasis expresivo, de la región de origen del hablante y del contexto interconsonántico, desde largas, semilargas, breves y superbreves, hasta llegar a desaparecer en algunos casos. Esta descripción que parece apuntar a que el marroquí, por poseer un sistema vocálico más complejo, no debería tener problemas con el sistema vocálico español, se contradice con la realidad, ya que uno de los rasgos más característicos de la pronunciación del español por los marroquíes es la impericia para dar con el timbre vocálico adecuado. La explicación se encuentra en que mentalmente, el marroquí tiene asumido el sistema vocálico del árabe escrito, que contiene únicamente el triángulo /a, i, u/, en cuanto al timbre se refiere. Así pues, para un marroquí, hay matices vocálicos en una misma palabra, que no constituyen un rasgo pertinente ni funcional, dado que no producen cambio en el significado. [...] Y luego, el alumno marroquí trasladará, inconscientemente, su pronunciación vocálica al español, apreciándosele como rasgos más característicos:

- la conversión de "e" en "i” y viceversa. Ejs.: /hago los diberes a las 5 y midia/ o, /¡mera!/ por “¡mira!”

- la conversión de "o” en "u” y viceversa. Ejs.: /bio la lona/ por "veo la luna” o /musaico/ por "mosaico".

- y la supresión de vocales en palabras largas. Ej.: /marroknería/ por "marroquinería”. 


\begin{tabular}{|c|c|c|}
\hline TIPO & REALIZACIÓN & CORRECCIÓN \\
\hline \multirow{12}{*}{$\mathrm{i}>\mathrm{e}$} & Recibedo (D) & recibido \\
\hline & Envitarme (D) & invitarme \\
\hline & Envitado (D) & invitado \\
\hline & en mediata (D) & inmediata \\
\hline & disponible (D) & disponible \\
\hline & envitacion (D) & Invitación \\
\hline & enterna $(A)$ & Interna \\
\hline & $\operatorname{vinir}(\mathrm{A})$ & venir \\
\hline & Vesitala (D) & visitarla \\
\hline & cosenar & cocinar \\
\hline & fenes $(\mathrm{A})$ & fines \\
\hline & Rodregue (A) & Rodrigo \\
\hline \multirow{7}{*}{$e>i$} & ci & que \\
\hline & hi & he \\
\hline & corio & correo \\
\hline & Porqui / porki & porque \\
\hline & in & en \\
\hline & agradisco & agradezco \\
\hline & vinir & venir \\
\hline \multirow{6}{*}{$\mathrm{U}>0$} & bosco & busco \\
\hline & so posto & supuesto \\
\hline & mocho & mucho \\
\hline & costaria & gustaría \\
\hline & complianos & cumleaños \\
\hline & pregonta & pregunta \\
\hline \multirow{4}{*}{$0>U$} & lusinto & lo siento \\
\hline & electrunico & electrónico \\
\hline & jurnada & Jornada \\
\hline & pur & por \\
\hline \multirow[t]{2}{*}{$e>0$} & confirmo & conforme \\
\hline & telofono & teléfono \\
\hline $0>\mathrm{e}$ & Rodregue & Rodrigo \\
\hline $0>\mathrm{i}$ & confirmo & conforme \\
\hline$a>e$ & ententamente & atentamente \\
\hline $0>a$ & trabaja & trabajo \\
\hline \multirow{3}{*}{ ie $>$ e } & experencia & experiencia \\
\hline & cento & ciento \\
\hline & tene & tiene \\
\hline \multirow{4}{*}{ ie $>$ i } & kiro & quiero \\
\hline & lusinto & lo siento \\
\hline & losinto & lo siento \\
\hline & exprinca & experiencia \\
\hline ie $>$ ei & seite & siete \\
\hline ie $>$ ia & limpiaza & limpieza \\
\hline ia $>a$ & exprinca & Experiencia \\
\hline io $>0$ & construccon & Construcción \\
\hline
\end{tabular}

Tabla 6. Errores en la realización de vocales, diptongos e biatos (Continúa) 


\begin{tabular}{|l|l|l|}
\hline \multirow{2}{*}{ ue $>0$} & Soposto & supuesto \\
\cline { 2 - 3 } & podo & puedo \\
\hline ue $>$ eu & peudo & puedo \\
\hline ui $>$ u & cudora & Cuidadora \\
\hline ui $>$ ue & cuedar & cuidar \\
\hline ui $>$ oi & moi & muy \\
\hline au $>$ a & restarante & Restaurante \\
\hline ai $>$ ae & traego & traigo \\
\hline ao $>$ aa & ahara & ahora \\
\hline ea $>$ ia & complianos & cumpleaños \\
\hline eo $>$ io & corio & correo \\
\hline
\end{tabular}

Tabla 6. Errores en la realización de vocales, diptongos e hiatos (Continuación)

En el caso de nuestros informantes, hemos recogido un total de 56 errores vocálicos (incluidos los hallados en diptongos) cuya distribución se puede observar la tabla 7.

\begin{tabular}{|l|l|}
\hline Confusión $\mathrm{i}>\mathrm{e}$ & 12 palabras (abertura del timbre) \\
\hline Confusión $\mathrm{e}>\mathrm{i}$ & 7 palabras (cierre del timbre). \\
\hline Confusión $\mathrm{u}>0$ & 6 palabras (abertura del timbre). \\
\hline Confusión $0>\mathrm{u}$ & 4 palabras (cierre del timbre) \\
\hline Otros & 6 palabras \\
\hline Diptongos & 21 palabras \\
\hline
\end{tabular}

Tabla 7. Total de errores cometidos por confusión del timbre de las vocales

Este resultado nos permite confirmar que se trata de errores persistentes, difíciles de solucionar. Y añadimos una nueva reflexión: Si, como hemos hecho más arriba, contrastamos los sistemas vocálicos del árabe y el español, podríamos deducir que, si bien el sistema vocálico del español es más complicado que el árabe, habría que pensar que esta complicación sólo afecta a los pares /e/, /i/ y /o/, /u/ lo que, por sí sólo, no debiera resultar tan problemático como se manifiesta.

El análisis acústico de las vocales españolas apoya esta tesis, pues los valores, en hercios, de cada uno de los dos primeros formantes: F1 (que señala la oposición alta/baja) y F2 (que señala la oposición anterior/posterior), están netamente diferenciados. 


\begin{tabular}{|c|c|c|}
\hline Vocal & Fl & F2 \\
\hline A & $700 \mathrm{~Hz}$ & $1460 \mathrm{~Hz}$ \\
\hline E & $360 \mathrm{~Hz}$ & $1970 \mathrm{~Hz}$ \\
\hline $\mathrm{I}$ & $248 \mathrm{~Hz}$ & $2600 \mathrm{~Hz}$ \\
\hline 0 & $400 \mathrm{~Hz}$ & $896 \mathrm{~Hz}$ \\
\hline U & $260 \mathrm{~Hz}$ & $613 \mathrm{~Hz}$ \\
\hline
\end{tabular}

Tabla 8. Análisis acústico de las vocales en español

Habría que pensar, por consiguiente, en otras causas añadidas. En primer lugar; si pasamos del plano de la fonología al de la fonética, el

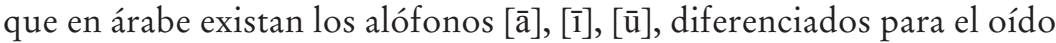
de los hablantes arabófonos, ¿facilitaría o dificultaría la asimilación de las oposiciones que sí se establecen en español; oposiciones del tipo: peso/piso, poso/puso...? Una deducción lógica sobre este sistema de breves y largas debería apoyar la tesis de que la facilitaría, pero los resultados ponen de manifiesto que la dificulta. Así lo piensa también Benyaya (2007:5):

Sin embargo, estos alófonos fácilmente perceptibles para el oído, no son fonológicamente pertinentes, de ahí la dificultad de su discriminación en el español. (...) En árabe, las vocales en contacto con las consonantes faríngeas y uvulares se ven influenciadas por éstas abriéndose, como es el caso del fonema [a] que suele realizarse como [x], e incluso como [e]. Éste es el origen de la confusión entre las vocales españolas a y e. Y teniendo en cuenta que en muchos casos, ésta es la única marca morfológica pertinente para expresar el modo en español, este problema puede llegar a ser muy peliagudo: [averigwáis] (por averigüéis), [sáis] (por seis), [bendéra] (por bandera), [balánsja] (por Valencia), [delaitár ] (por deleitar)

Al problema de la oposición entre vocales medias y altas, habría que añadir el de la discriminación entre a y e, por asimilación cuando entran en contacto con una consonante faríngea o uvular. Y concretándolo en el caso del dialecto marroquí, afirma Benyaya que el fenómeno de la reducción de la vocalización es más drástico que en los dialectos árabes orientales, hasta llegar a producirse vocales muy breves o con tendencia a la completa desaparición. Esto puede explicar que hayamos encontrado ejemplos de síncopas vocálicas como: [eltrónico] por electrónico, [vóstros] por vosotros, si bien en número no suficientemente significativo como para establecer una generalización. Benyaya pone algunos ejemplos más radicales: [brwén_a] por vergüenza, [prspktíva], por perspectiva, [miditránjo] por Mediterráneo. Estas afirmaciones nos llevan a pensar que no resulta nada fácil, ni para enseñantes ni para aprendientes, dar con fórmulas útiles que sirvan para ajustar una mentalidad acostumbrada a un sistema fonológico de base consonántica a un nuevo sistema de base vocálica. Desde los ini- 
cios del aprendizaje del español (también el francés), se hace necesaria una seria reflexión sobre la sílaba pues, si en español (como en francés), la vocal es el elemento central de la sílaba, en árabe lo son las consonantes. Y sigue diciendo que el árabe clásico no admite muchas combinaciones silábicas pero que el dialectal es mucho más flexible por lo que, según él, la silabización española no le entraña excesiva dificultad, salvo algunos grupos consonantes como "sc" o "xc", en palabras como escena o excelente con los que se tiende a la simplificación: essena, eccelente. Los datos obtenidos no apoyan esta afirmación de Benyaya. Hamparzoumian y Barquín (2005), después de hacer una comparativa entre las combinaciones silábicas del español y el árabe, deducen que el encabalgamiento de consonantes o la formación de sílabas complejas no tiene por qué causar problemas a los marroquíes a no ser por el emparejamiento de algunas consonantes concretas de difícil pronunciación como:"cz"; ej.: pizca, "sc"; ej.: escena, "pl"; ej.: plato... pero, analizado el fenómeno, llegan a la siguiente conclusión, que nos parece significativa:

Algunas prácticas didácticas poco eficaces provocan que el alumno no logre suficiente pericia en este sentido, lo que sería fácilmente subsanable presentando ejemplos de paralelismos entre las dos lenguas. Las encuestas revelan cierta falta de conciencia, por parte de los profesores, sobre estos problemas, o, tal vez, el trabajo con los niños no sea lo suficientemente específico como para su detección y la implementación de recursos didácticos para solucionarlos por el momento. Hamparzoumian y Barquín (2005:36)

Por nuestra parte, añadimos otra causa más: venimos advirtiendo que, conforme al sistema educativo marroquí, los escolares experimentan el primer contacto escolar con otra lengua extranjera, que es el francés. Los dos primeros sistemas que contrastan entonces son árabe y francés. El sistema fonológico de las vocales del francés, comparado con el del español y lógicamente con el árabe, es de mucha mayor complejidad, como se demuestra en la tabla 8. 


\begin{tabular}{|c|c|c|c|}
\hline & \multicolumn{3}{|c|}{ Punto de articulación } \\
\hline & \multicolumn{2}{|c|}{ Anteriores } & Posteriores \\
\hline & No labializadas & Labializadas & \\
\hline Cerradas & $\begin{array}{l}\text { /i/ } \\
\text { ville, île, naîf, cyprès }\end{array}$ & $\begin{array}{l}\text { /y/ } \\
\text { bossu, mûr }\end{array}$ & $\begin{array}{l}\text { /u/ } \\
\text { bout, dégoût }\end{array}$ \\
\hline Mediocerradas & $\begin{array}{l}\text { le/ } \\
\text { thé, fée }\end{array}$ & $\begin{array}{l}\varnothing / \\
\text { deux, voeu }\end{array}$ & $\begin{array}{l}10 / \\
\text { dos, chaude, beau }\end{array}$ \\
\hline Medioabiertas & $\begin{array}{l}\text { /\&/ } \\
\text { net, père, fête } \\
\mid \tilde{\varepsilon} / \\
\text { imbécile, brin, examen }\end{array}$ & 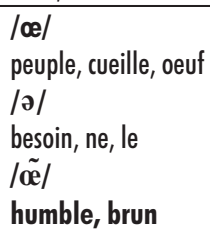 & $\begin{array}{l}/ \mathrm{o} / \\
\text { coq, rougeole } \\
\text { /õ/ } \\
\text { bon, nom, jungle }\end{array}$ \\
\hline Abiertas & $\begin{array}{l}\text { /a/ } \\
\text { patte, là }\end{array}$ & & $\begin{array}{l}\text { /â/ } \\
\text { pâte, las } \\
\text { /ã / } \\
\text { banc, champ, temps }\end{array}$ \\
\hline
\end{tabular}

Tabla 9. Sistema vocálico del francés

Si un estudiante comienza el estudio del español con un nivel de francés suficiente, cuando ya ha estudiado este idioma durante 4 cursos de Primaria y 3 cursos de Secundaria, parecería lógico que la inmersión en el sistema fonológico de las vocales del español como L3 fuera senci1la. Los resultados del análisis, tanto cuantitativo como cualitativo de las muestras, no justifican tal situación, lo que induce a pensar:

1. Que la competencia que han adquirido en la L2, hasta ese momento, no goza de suficiente solidez como para hacer de puente hacia una transición cómoda a la L3.

Chami y El Bekraoui (1991:46-47) llegan a la siguiente conclusión en su trabajo:

Constater- et déplorer- une baisse progressive et quasigénéralisée du niveau en français dans l'ensemble du cursus scolaire marocain est certes sans originalité. Les résultats tiennent pour une grande part au mode d'application des instructions officielles : niveau de formation des maîtres, capacité à intégrer de nouvelles pédagogies, aptitude à passer de la théorie à la pratique réelle des classes. Les concepts, et les consignes, de compétence communicative, énonciation, actes de parole, stratégies individuelles ont succédé aux instructions de 1978, davantage orientées vers le montage d'automatismes par des exercices structuraux. Mais des difficultés subsistent et s'aggravent, en particulier pour les élèves issus de milieu socialement et culturellement défavorisés. Seule une forte motivation dans l'apprentissage du français semblerait capable d'inverser ce mouvement de déclin, par une revalorisation de cette matière à la fois à l'intérieur du système scolaire (par les coefficients et les notes éliminatoires) et dans le réseau des échanges économiques et culturels, si la langue française demeure un véhicule de savoir et de développement. 
2. Que los estudiantes marroquíes sienten que el español es una lengua próxima al francés y consideran que, para hacerse comprender en esta lengua, pueden hacerlo con los conocimientos adquiridos en francés y conceden escasa o nula importancia a interesarse por los hechos diferenciales de la L3 respecto a la L2, por ende, mal aprendida.

3. Que la mayoría de los errores podemos achacarlos, más que a elementos del sistema mal asumidos, a los llamados errores fonéticos esporádicos, que actúan como universales lingüísticos y que son los principales responsables de la evolución fonética de las lenguas.

4. De los 12 casos de transformación $i>e, 4$ se deben a un fenómeno de asimilación: fenes, en mediata, enterna, vinir y 7 al de disimilación: envitarme, envitado, desponible, envitación, vesitala, recibedo, Rodregue. De los 6 casos de transformación u>o, 4 son achacables a la asimilación: bosco, soposto, mocho, complianos.

5. Por lo que respecta a los diptongos, resultan ser un fenómeno desconocido para los hablantes marroquíes a pesar de que, como señala Benyaya (2007:7), las consonantes /waw/ y /yae/ tienen el carácter de semiconsonantes, vocalizadas, y resultan fonológicamente muy similares a los diptongos en español, pero como no se trata de un elemento digráfico, el diptongo es difícilmente perceptible para el alumno arabófono, que tiende con frecuencia a reducirlo a una vocal simple. De los 21 errores registrados, 13 responden a esta tendencia de reducción y 8 suponen una modificación del diptongo por otra secuencia digráfica vocálica, sin que hayamos podido observar ninguna tendencia generalizable.

\section{Conclusiones}

A modo de conclusión, los hallazgos del presente estudio han revelado que los errores gráficos son el principal escollo con que se enfrentan nuestros informantes a la hora de redactar textos en español. Hemos comentado, en el apartado precedente, una amplia casuística de dificultades (ortográficas, fonéticas y morfosintácticas) muy arraigadas en la escritura de los alumnos marroquíes, principalmente por interferencia de las LM y L2, que también provienen de la lengua oral. La barrera que impide un correcto desarrollo de sucesivos estados de interlengua escrita puede ser por la falta de práctica de escritura de textos en general. Nos atrevemos a aventurar que estas dificultades paralizantes consisten en no haber asimilado desde un principio una distinción básica: el hecho de que la sílaba en español (como en francés) es nuclear vocálica, a diferencia de la estructuración consonántica de la sílaba árabe. Así parecen apoyar este criterio las opiniones de Benyaya (2007) y de Hamparzoumian y Barquín (2005), quienes contrastan las distintas estructuras silábicas del español con las del árabe, dejando claro que 
el español tiene una mayor complejidad silábica que el árabe culto, si bien, en el dialecto marroquí, se dan bastantes más combinaciones que en el árabe estándar. En consecuencia, se trata de un problema de prosodia. La confección de baterías de ejercicios orales y escritos, bien programados, para garantizar una adquisición progresiva del objetivo que pretendemos, ejercicios adecuados de lectura y entonación, podrían servir para atenuar, primero, y salvar, más adelante, el escollo que supone el grave problema que el estudiante marroquí de español tiene para familiarizarse con conceptos para él tan abstrusos como acento de intensidad, grupo fónico, curva melódica entonativa, pausa entonativa y pausa gramatical. Familiarizarse, dicho sea de paso, de manera intuitiva y práctica, ni teórica, ni científica. Las limitaciones de la extensión exigida para un artículo como en el que nos ocupamos, no permiten la posibilidad de desarrollar aquí los principios metodológicos mediante una programación de objetivos, selección de contenidos y propuesta de actividades, ya que sobrepasaríamos los límites de la intención de nuestro estudio. Una vez conseguido, facilitaríamos, de manera intuitiva, insistimos, la distinción entre las aperturas media y alta de las vocales, la discriminación de las sílabas tónica y átonas en la palabra y la función de la ortografía de los signos de puntuación. Podemos concluir también que nuestros informantes usan sus conocimientos de francés a la hora de expresarse en español, lo que representa antes de todo una gran ventaja por la proximidad y las transferencias positivas. En la opinión compartida por El-Madkouri y Soto (2009) y Benyaya (2007:13), el español, para los marroquíes, es "muy parecido al francés con ligeros cambios: para muchos inmigrantes, el mero hecho de saber francés presupone la no necesidad de aprender el español de forma reglada". Ahora bien, estos aprendientes multilingües producen también errores de transferencia negativa en su lengua escrita por lo que sería necesario insistir en esas dificultades a la hora de formarlos. A la luz del análisis de las producciones escritas de nuestros informantes, hemos detectado una serie de fenómenos frecuentes y de estrategias comunes que se producen en todo aprendizaje tratándose principalmente de errores interlingüísticos. En el plano fonológico, se manifiesta claramente la influencia del árabe a la hora de confundir o neutralizar algunas vocales. En el plano morfosintáctico, esta interferencia se manifiesta también en el uso del sistema verbal y en algunas estructuras, calcos del árabe. Hemos visto también que la interferencia con su segunda lengua -en este caso el francés- constituye, asimismo, una referencia ineludible y se manifiesta como una constante fuente de interferencias positivas y negativas, en todos los niveles de la lengua. Pensamos que es importante que el docente sepa aprovechar estos conocimientos y las semejanzas francés-español, pero también que sepa fijar la atención sobre las posibles transferencias negativas, para ayudar al alumno marroquí en el proceso de adquisición o aprendizaje de la lengua española. 
Sería inoportuno que, como ya hemos adelantado en el apartado 3.2, termináramos sin recordar que nuestras conclusiones, por las limitaciones de nuestro estudio, no pueden ser consideradas como caracterizadoras de un determinado nivel de interlengua que pudiera entenderse extrapolable. Creemos, no obstante, haber puesto de manifiesto el panorama mostrado por esa población de origen marroquí que pretende certificar una cualificación en español, suficiente para su integración lingǘstica en nuestro idioma. Algunos de nuestros informantes lo consiguieron, otros no. Como quiera que sea, la tarea no debiera acabar en este punto y quisiéramos haber contribuido con nuestro artículo a clarificar tal panorama. Es nuestro deseo.

Anna Doquin de Saint Preux

Universidad Antonio de Nebrija

Correo: adoquins@nebrija.es

ORCID: 0000-0002-6196-7608
Cristina Mata Verdoy

Consejería de Educación de Embajada de España en Francia

Correo: crismaver@gmail.com

ORCID: 0000-0002-4068-0800

Recepción: 22/11/2018; Aceptación: 15/09/2020

\section{Notas}

1 Madkouri 1995 llevó a cabo sus investigaciones mediante el estudio de grabaciones de conversaciones de interacciones comunicativas informales entre aprendices marroquíes, adultos y niños, e interlocutores nativos, tanto en contextos naturales de adquisición como en contextos mixtos (académicos) de aprendizaje.

2 Benyaya 2007 para la elaboración del corpus, procedió a la realización de varias grabaciones en distintos institutos públicos ubicados en diferentes ciudades y regiones de Marruecos con alumnos de edades comprendidas entre 16 y 18 años, pertenecientes a los niveles elemental, medio y avanzado, correspondientes al Ciclo de Cualificación (Tronco Común), y a primero y segundo de Bachillerato, respectivamente), a partir de diversas pruebas: de conversación espontánea, de lecturas controladas, a partir de una selección de textos elaborados ex profeso, una prueba de dictado, amén de una amplia recopilación de exámenes realizados por estos mismos alumnos a lo largo del curso 2004-2005, en calidad de controles continuos evaluables, de acuerdo con lo que estipula la normativa por la que se rige la secundaria marroquí, y que constan de un bloque de preguntas de comprensión, de un bloque de gramática, y finalmente de una prueba de redacción.

3 Las opiniones de Ammadi se han tomado desde Addouch 2013.

\section{Referencias bibliográficas}

Addouch, B. 2013. Las unidades fraseológicas en la enseñanza del español (caso del alumnado marroqui del Instituto Cervantes de Tetuán). Tesis doctoral. Universidad Mohammed V-Agdal.

Al-Madkouri, Mohamed. 1995. "La lengua española y el inmigrante marroquí". Ediciones Complutense. Didáctica. Lengua y literatura, 7. 355-362.

Al-Zawan, Khaled Omran. 2005. Análisis de errores en el aprendizaje del español como lengua extranjera de estudiantes universitarios árabe. Tesis doctoral: Universidad de Alicante.

Lengua y migración / Language and Migration 12:2 (2020), 109-131

Edición impresa: ISSN 1889-5425. Edición en línea: ISSN 2660-7166. @) Universidad de Alcalá 
Ammadi, Mostafa. 2009. Dificultades específicas en la enseñanza del español a marroquies. Rabat: Bouregreg.

Benyaya, Zineb. 2007. "La enseñanza del español en la secundaria marroquí: aspectos fónicos”. Revista Nebrija de Lingüistica Aplicada a la Enseñanza de Lenguas, 1: 2.1-60.

Berengueras, Mercè. 2013. "El sistema educativo de Marruecos. Avances en Supervisión Educativa”, Dialnet, 19. Universidad de Rioja.

https://avances.adide.org/index.php/ase/article/view/122

Chami, Félix y El Bekraoui, Naïma. 1991. "L'enseignement du français au Maroc : Démarches pédagogiques - Essai d'évaluation”, L'Information Grammaticale, 51 : 44-47.

Doquin de Saint Preux, Anna. y Sáez, Patricia. 2014. "Errores gramaticales persistentes en la Interlengua de estudiantes francófonos de ELE de nivel superior”. MarcoELE, revista de didáctica ELE, 19: 1-18

El-Madkouri, Maataoui. 1995. "La lengua española y el inmigrante marroquí”. Didáctica, 7. 355-362.

El-Madkouri Maataoui y Soto, Beatriz. 2009. “Aproximación pluridimensional a la influencia interlingüística: La adquisición del español como lengua de acogida en población marroquí inmigrada en España”. SLEi en red: Segundas Lenguas e Inmigración en red, 2. 3-37 https://dialnet.unirioja.es/descarga/articulo/3186917.pdf

Fernández, Sonsoles. 1997. Interlengua y análisis de errores en el aprendizaje del español como lengua extranjera. Madrid: Edelsa.

Hamparzoumian, Aram y Barquín, Javier. 2005. “La Competencia Lingüística de los niños marroquíes escolarizados en Andalucía”. Aldadis.net, 4. 30-54 http://www.aldadis.net/revista4/04/imagen/05aram.pdf

Moscoso, Francisco. 2003. "Situación lingüística en Marruecos: árabe marroquí, beréber, árabe estándar, lenguas europeas”. Al-Andalus-Magreb, 10. 167-186.

Santos Gargallo, Isabel. 1993. Análisis contrastivo, análisis de errores e interlengua en el marco de la lingüística contrastiva. Madrid: Síntesis.

Santos de la Rosa, Inmaculada. 2014. "Aspectos básicos del análisis de errores de alumnos arabófonos para profesores de enseñanza de español. Investigaciones sobre la enseñanza del español y su cultura en contextos de inmigración”. Investigaciones sobre la enseñanza del español y su cultura en contextos de inmigración [archivo de ordenador], 189-220.

https://dialnet.unirioja.es/servlet/articulo?codigo $=4966049$

Sáez, Patricia. 2012. Errores gramaticales en las producciones escritas y orales de aprendices franceses de ELE de nivel C1. Trabajo Fin de Máster. Universidad Antonio de Nebrija.

Vázquez, Graciela. 1999. ¿Errores? ¡Sin falta! Madrid: Edelsa. 